\title{
Targeted nanotechnology: delivering small but deadly punches
}

\section{Editorial}

The traditional drug delivery methods, which basically include the Oral and IV routes of administration, are still the most widely used today, yet each has its disadvantages. Oral delivery via tablets or capsules is largely inefficient due to exposure of the pharmaceutical agent to the metabolic processes of the body. Therefore, a larger than necessary dose is often required and the maximum effectiveness of the drug is limited. Traditional intravenous (IV) administration is even much more problematic. Specificity for IV injectable drugs is often low, necessitating large amounts of a drug be injected into a patient, creating a high concentration of the drug in the blood stream that could potentially lead to toxic side effects.

Throughout various fields of science and technology, a push towards the use of nano-scale technology is well underway. Nanotechnology has the potential to produce low-cost, self-replicating systems that could revolutionize the scientific landscape. Nanoparticles are generally defined as particles between 10nanometers (nm) and $1000 \mathrm{~nm}$ in size.

One area where nano-scale work is already well underway is within the field of drug delivery. In drug delivery, nanoparticles are fabricated in order to entrap and deliver specific pharmaceutical payloads to various locations within the body. Nanoparticles are subjected to state-of-the-art engineering in order to increase their affinity for diseased cells, which will culminate in the treatment of the cells. This technique reduces damage to healthy cells in the body. For example, tests are in progress for targeted delivery of chemotherapeutic drugs and their final approval for use in cancer patients. CytImmune, a clinical stage biopharmaceutical company based in Rockville, MD, USA, has published the preliminary results of a Phase 1 Clinical Trial of their first targeted chemotherapy drug and another company, BIND Biosciences, has published preliminary results of a Phase 1 Clinical Trial for their first targeted chemotherapy drug and is proceeding with a Phase 2 Clinical Trial. Researchers at MIT are using nanoparticles to deliver vaccine. The nanoparticles protect the vaccine, allowing the vaccine time to trigger a stronger immune response. The same group has developed a nanoparticle that can be taken orally and pass through the lining of the intestines into the bloodstream. This should allow drugs that must now be delivered with a shot to be taken in pill form.

Nanotechnology is also being applied to cancer in two broad areas: the development of nanovectors, such as nanoparticles, which can be loaded with drugs and then targeted to tumours, and high-throughput nanosensor devices for detecting the biological underpinnings of cancer. Taken together, such technologies could lead to better treatment for patients with cancer.

One of the key challenges in targeted nanotechnology is targeting them to appropriate tissues and cells. Extensive work has been done on Polymeric nanoparticles as potential drug delivery systems for many years, particularly, on how these nanoparticles can be delivered to various tissues following intravenous administration. Early experiments demonstrated that nanoparticles are normally taken up by macrophages responsible for protecting the body from
Volume I Issue I - 2014

\author{
Stanley Moffatt \\ School of Informatics, Health and Allied Sciences, Regent \\ University College of Science and Technology, Ghana
}

\begin{abstract}
Correspondence: Stanley Moffatt, School of Informatics, Health and Allied Sciences, Institutional Advancement, Regent University College of Science and Technology, Ghana, Tel +233242169839, Email stanley.moffatt@regent.edu.gh
\end{abstract}

Received: April 29, 2014 | Published: April 30, 2014

invading bacteria and viruses, and that this uptake could be prevented by appropriate nanoparticle surface coatings. Subsequent work from different laboratories showed that more subtle variations in coatings and their interaction with biological components can lead to a wider range of bio-distributions.

The basis of tumor-specific targeting is that a drug conjugated to a tumor-specific molecule will remain inactive until it reaches the tumor. Although targeting with conjugates like antibodies, has been an option of choice, targeting of these nano-vectors to precise locations in the cell has even become more relevant. In this regard the physical characteristics of the particles play an essential role. Mauro Ferrari, a professor of nanotechnology at the University of Texas Health Science Center, the M.D. Anderson Cancer Center, and Rice University in Houston, USA says, "The size, shape, physical properties, density and charge all affect how particles travel through the body, and whether or not they will cross biological membranes". Dr. Mauro and his team have done lots of work to empirically demonstrate that cellular barriers determine the distribution and location of injected nanoparticles in the body, and that a very careful and analytical selection of choice, size and shape of the nano-vector can enhance the amount of drug delivered to the target site. These targeting systems would ideally be dependent on interactions with cells found specifically on the surface of tumor cells and not the surface of healthy cells. Many experts however believe that these therapies using antibodies directed to cancer targets will dominate the market for the foreseeable future. While these antibodies can prove to be therapeutic agents in their own right, they also have the ability to serve as carriers for drug delivery systems for even more effective and less intrusive cancer therapy.

One other strategy is tumor vasculature targeting, and this can allow targeted delivery to a wide range of tumor types. The first vascular targeting was approved by the FDA in 1999 for treatment of age-related muscular degeneration. In 2003, clinical trials with the antiangiogenic drug, AvastinR (Genentech), showed that its use can prolong survival in patients with metastatic colorectal cancer. Indeed, recent works by us and others have also shown that gene delivery may also be targeted to neovasculature by coupling cationic nanoparticles to an integrin-targeting ligand in tumor-bearing mice. Perhaps most significantly in the results, no gene expression was detected for targeted particles injected with an excess of a control scrambled ligand, 
implying that the ligand was selectively bound to the tumor over the ligand-nanoparticle combination and fully blocked the particles from reaching the tumor. Mice that were treated with PBS control, empty targeting nanoparticles or loaded targeting nanoparticles injected with an excess of targeting ligand showed no signs of a slowing of tumor growth and had to be killed after three weeks. However, those mice injected with nanoparticles carrying the targeting gene showed a significant regression in tumor size, which was sustained over an appreciable number of days.

The utilization of cutting-edge proteomics in fabrication technologies to produce shape-specific organic nanoparticles in order to synthesize truly engineered particles with appropriate characteristics has also paid great dividends in targeted nanotechnology. Indeed, utilization of proteomics in this regard allows precise control over particle size $(20 \mathrm{~nm}$ to $>100 \mu \mathrm{m})$, particle shape (spheres, cylinders, discs, toroidal), particle composition (organic or inorganic, solid or porous), particle cargo (hydrophilic or hydrophobic therapeutics, biologicals, imaging agents), particle compliance (stiff, deformable) and particle surface properties (Avidin-biotin complexes, targeting peptides, antibodies, aptamers, PEG chains). Some proteomics studies have even gone further to show that red blood cells can be manipulated to produce compliant nanoparticles that can deform in order to cross biological barriers in the spleen or the blood-brain barrier.

As novel tumor-targeted nanotechnology products move from the laboratory to the clinic, some of the limitations existing due to the lower potency of some drugs after being linked to targeting moieties, when the targeting portion is not cleaved correctly or at all, need urgent attention. Nanoparticles can be inhaled, ingested or absorbed through the skin, and they can penetrate cells, even into the cell nucleus, where, if sufficiently small, they can come into close contact with genetic material. Consequently, nanotoxicology, which is the study of the potential negative impact of the interactions between nanomaterials and biological systems, has evolved. ${ }^{1}$ Some preliminary nanotoxicity investigations have led to the speculation that nanomaterials may contribute to the damage of brain cells ${ }^{2}$ and other areas of the body that are more susceptible to toxic effects. ${ }^{3}$ Several mechanisms have also been proposed to affect the toxicity of nanomaterials, depending on their physiochemical properties and environmental conditions. For example, naked quantum dots show cytotoxicity induces reactive oxygen species, thereby damaging the nucleus, mitochondria and plasma membranes. ${ }^{4}$ For silica nanoparticles, only concentrations above $0.1 \mathrm{mg} / \mathrm{ml}$ have been found to be toxic. ${ }^{5}$ In addition, it was reported that most cationic nanoparticles can cause hemolysis and blood clotting, while neutral and anionic nanoparticles are quite nontoxic. ${ }^{6}$

Further, the issue of regulatory approval of these new technologies will also come to the fore. There are several issues under discussion in various forums related to the FDA's regulation of nanotechnology products. FDA expects many regulated nanotechnology products to span the regulatory boundaries between pharmaceuticals, medical devices and biological systems. As new toxicological risks that derive from the new materials and/or new conformations of existing materials are identified, new tests will be required. Since FDA regulates products, and not technology, the need for a continuous and systematic upgrade of existing technologies to churn out more efficacious nanotechnology products cannot be overlooked.

Research activity aimed towards achieving specific and targeted delivery of anti-cancer agents has expanded tremendously in the last few years with new avenues of directing drugs to tumors as well as new types of drugs. However, there is a great deal more that can be done to treat and perhaps prevent advanced cancer, simply by treating it in the early stages. Nanomaterial toxicity should be considered relative to the patient population, as well as the entire manufacturing and disposal processes. Based on safety concerns, the establishment of standards or reference materials and consensus testing protocols that can constitute benchmarks for churning out novel classes of nanomaterials are needed. This will obviously require superior targeting methods which many researchers will undoubtedly pursue and hopefully achieve, to further leverage the full benefits of targeted nanotechnology.

The field of nanotechnology is moving forward rapidly. There is no doubt that it will enhance our understanding of biology and how biological systems work. In particular, multifunctional therapeutics where a nanoparticle serves as a platform to facilitate its specific targeting to cancer cells and delivery of a potent treatment, minimizing the risk to normal tissues is a medical revolution. In the future, nanotechnology will enhance the drug discovery process, through miniaturization, speed and reliability of assays. It will also allow greater selection of the right drug for the right patient and enable the tests to support this decision process to be done in the doctor's clinic.

\section{Acknowledgements}

None.

\section{Conflict of interest}

The author declares no conflict of interest.

\section{References}

1. Jiang W, Kim BYS, Rutka JT, et al. Nanoparticle-mediated cellular response is size-dependent. Nature Nanotechnology. 2008;3:145-150.

2. Thrall L. Study links TiO2 nanoparticles with potential for brain-cell damage. Environ Sci Technol. 2006;40(14):4326-4327.

3. Oberdorster G, Oberdorster E, Oberdorster J. Nanotoxicology: an emerging discipline evolving from studies of ultrafine particles. Environ Health Perspect. 2005;113(7):823-839.

4. Lovric J, Bazzi HS, Cuie Y, et al. Differences in subcellular distribution and toxicity of green and red emitting $\mathrm{Cd}$ Te quantum dots. $J$ Mol Med (Berl). 2005;83(5):377-385.

5. Chang JS, Chang KL, Hwang DF, et al. In vitro cytotoxicity of silica nanoparticles at high concentrations strongly depends on the metabolic activity type of the cell line. Environ Sci Technol. 2007;41(6):2064-2068.

6. Jong WHD, Borm PJ. Drug delivery and nanoparticles: applications and hazards. Int J Nanomed. 2008;3(2):133-149. 